\title{
The Design of Large Arc-shape High-precision Walking Device in TV (thermal-vacuum) Conditions
}

\author{
Xue Lian ${ }^{\text {a }}$, Nan Hua, Liu Jiaqi, Liu Xin and Meng Gang \\ National Key Laboratory of Science and Technology on Test Physics \& Numerical Mathematics
}

\begin{abstract}
The large-sized space environmental simulation test facility is mainly used for providing thermal radiation environment in high-vacuum, cold and dark space for satellites, spacecraft, lunar spacecraft to carry out whole satellite thermal vacuum test and for large equipment like antenna to carry out tests in TV conditions. Monitoring the spacecraft's surface optical image or temperature is a main task in space environment simulation test. In previous tests, optical image test mainly took place in a fixed position, so the measuring location and angle are limited. This paper focuses on large spherical space environmental simulation test facility, and designs a large arc-shape high-precision walking device in a space environment simulation test device. It introduces key technology in detail like structure design, thermal design, processes and manufacturing, etc. The test results show that this device can work steadily and reliably under simulation space environments, and the precision exceeds $0.5^{\circ}$.
\end{abstract}

\section{Introduction}

During the development of the spacecraft, in order to ensure correctness of spacecraft design and enhance reliability of on-orbit operation, it's a must to simulate cold and dark environments on the ground with solar radiation after the spacecraft is on the orbit, and carry out enough verification tests under such environments. The large-sized space environmental simulation test facility provides on-orbit environment simulation test conditions for aircrafts. Monitoring the spacecraft's surface optical image or temperature is a main task in space environment simulation test. In previous tests, optical image test mainly took place in a fixed position- - observing through optical window outside the space environmental simulation test facility or in a fixed position inside space environmental simulation test facility — - so the measuring location and angle are limited. Therefore, we need to design a walking device that can work in space environmental simulation facility, allow large-range movements of the measuring instrument and adjust the vision direction of the measuring instrument. However, the emphasis and difficulty in the research are put on the structure design, thermal design, process design and other key elements of the large movement equipment that can work reliably in TV conditions [1].

\section{Structure and Working Principles}

\footnotetext{
${ }^{a}$ Corresponding author : casc_xuelian@163.com
} 
The device consists of large arc-shape guide rail, track car, two-dimensional turntable, cable lead-out equipment and monitor system (including monitor software). The device's main body works in a large spherical space environmental simulation test facility $(\Phi 18 \mathrm{~m})$, and the structure is shown in Figure 1 below.

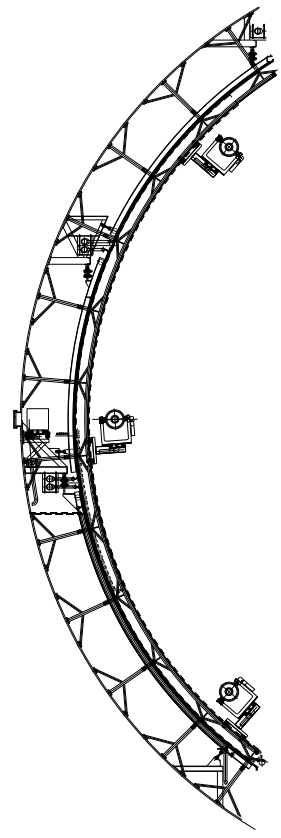

Figure 1. System Structure

The arc-shape guide rail permits the measuring instrument to rotate around the measuring aircraft, so as to do optical image measurements in different locations and at different angles. Two-dimensional turntable can enable test instruments to aim accurately. It's equipped with electric walking and posture adjustment to position rapidly and accurately. Considering that this device works in TV conditions, the design option should meet requirements of low temperature $(\leqslant 100 \mathrm{~K})$ and high vacuum $\left(\leqslant 10^{-3} \mathrm{~Pa}\right)$.

The Arc-shape track should be equipped in the longitude direction of the spherical space environmental simulation test facility, with a span of $500 \mathrm{~mm}$ between two tracks, an angle of $110^{\circ}$ between both ends of rail and the center of the circle, and single arc-shape track length of $15 \mathrm{~m}$. The arc-shape track is equipped with cable drag chain, and it can drive required power cables and signal cables to move with measuring instruments.

\section{Device Option}

\subsection{Arc-shape Guide Rail}

Arc-shape guide rail provides the track for the track car. It consists of arc-shape track, track support, track limiter, and rack, etc. The track is fixed in twelve supports (angle: $10^{\circ}$ ) preset on the tank. Major parts of the guide rail are weldments designed with guide rails and arc-shape racks. All are made of stainless steel for ultra-low temperature.

Under normal temperature and normal pressure, all parts of the track can meet precision requirement by machining processing. However, because of working in TV conditions, materials may contract when cold and cause the whole track to change shape, which influences precision greatly. In 
order to decrease the influence of track's thermal contraction, the track design needs to consider necessary compensation process.

\subsubsection{Compensation Process Design for the Track's Thermal Contraction}

The Arch-shape track's installation precision on the environmental simulation test facility guarantees precision of measuring equipment. The main track compensation processes are as below:

1) The track is designed to be stretchable in the longitudinal direction. Fixing the position of the track on the longitude near the equator can let it stretch out and draw back freely along the longitude.

2) The track is divided into several sections during processing, and they are connected with bolts on site with certain stretching gaps for each section, so as to offset thermal contraction and prevent tracks' coming off.

\subsubsection{Compensation Process Design for Track Supports' Thermal Contraction}

The Track Support connects the arc-shape tracks and the wall of the test facility, and is the structural support of whole arc-shape track. In the facility, there is liquid nitrogen heat sink, which can cause a severe drop in the environment temperature. By means of simulation, under low-temperature working conditions, the minimum limit will be about $175 \mathrm{k}$, and the temperature of areas connecting with the tank wall is close to normal temperature. There is a big temperature difference, and it leads to huge local deformation.

For a loaded track, shear stress on the support is the main load and is the most demanding for the joints, so the load capacity of joints should be enhanced. deformation in Figure 2. The biggest force bearing point is the joint with the pipe wall, where reinforcement is necessary.

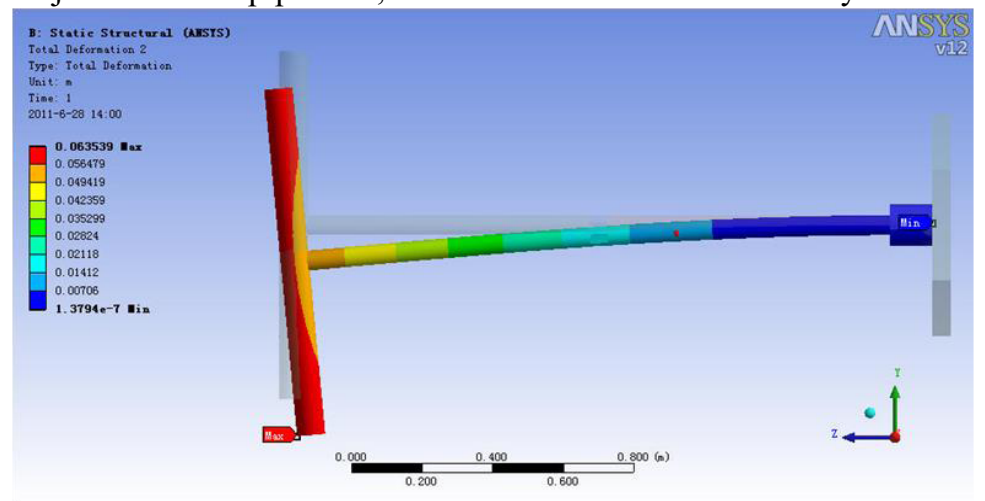

Figure 2. Deformation of the Whole Support

Regarding the value of deformation caused by temperature reduction and load, when there is a load, the track surface has a very big value of deformation up to $63 \mathrm{~mm}$. So side supports should be added with the structure support design in Figure 3 below. It can decrease some deformation effectively. The track supports are arranged symmetrically and evenly, so they can balance the stress and reduce local deformation. According to calculation results of stress analysis, there is more stress on the joints, so they should be made of stainless steel, reinforced with flanges, and equipped with shorter support bars. 


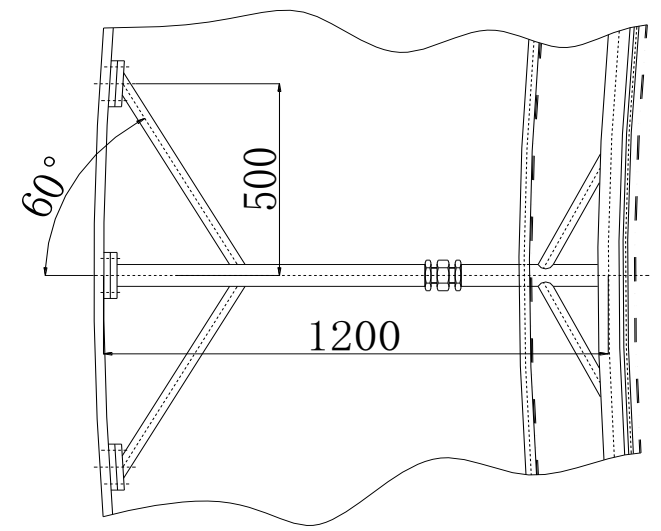

Figure 3. Arch-shape Track Support Structure

Main compensation processes for track supports include:

1) Use flanges to connect guide rails and the wall of the environmental simulation test facility. The arch-shape surface of the interior wall of the facility track is the installation base surface. However, there is dimension error in the arc-shape surface during production. When flanges are used for connection, gaskets can be added to adjust installation surfaces of the track to ensure the position tolerance between the track and installation base surface;

2) The connection rod of the track support is designed according to sections. In the middle there is adjustment equipment. The track's position tolerance is regulated by adjusting the screw nuts in the middle of the support.

3) The track support will not touch heat sink as there is a PTFE gasket in the middle. This can avoid heat leakage caused by heat conduction and too low local temperature of the track.

\subsubsection{Compensation Process Design for Racks' Thermal Contraction}

Rack is the transmission equipment for the whole device. Out-of-chain phenomenon should be avoided when the rack deforms due to thermal contraction. To avoid it, following compensation measures should be taken in the design:

1) The rack should be designed to be stretchable in the longitudinal direction. Fixing the position of the rack on the longitude near the equator can let it stretch out and draw back freely along the longitude. The fixation method is same with that of the arch-shape track.

2) Install the rack on the track support. Adjust the radius of the installation base of the rack through drilling slotted hole on the track. See Figure4.

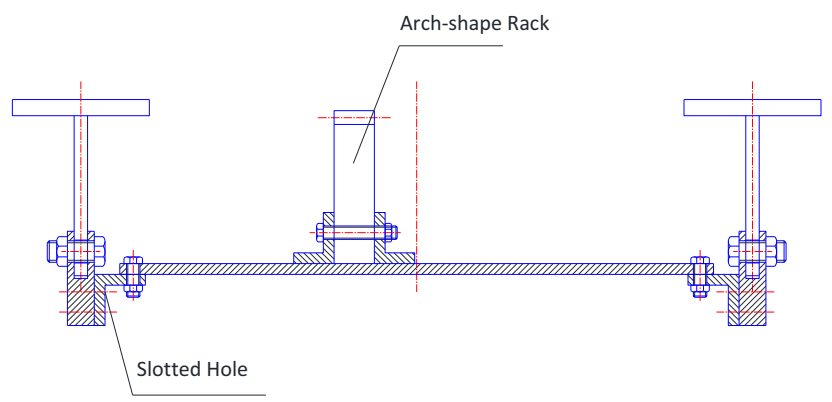

Figure 4. Installation Methods of Arc-shape Rack 
3) The rack is divided into several sections during processing, and they are connected with bolts on site with certain stretching gaps for each section and in the way of slanted plane intersection, so as to offset thermal contraction and prevent racks' coming off.

\subsection{Track Car and Two-dimensional Turntable}

The track car carries the two-dimensional turntable to move on the arc-shape track. The car is equipped with guiding wheels, driving motor, reducer and driving gear, etc. Use an imported right-angle precision gear reducer and carry out dry lubrication for the reducer [2], in order to meet requirements of working at high vacuums. Cable of the measuring instrument is fixed on the arc-shape track car by hanging, and going into the drag chain with equipment cables. See the structure in Figure 5 .

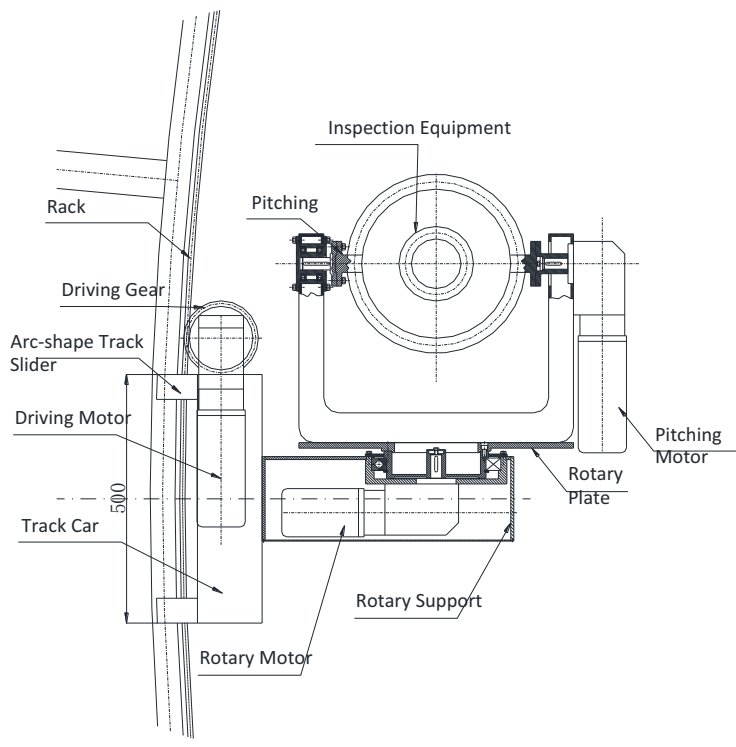

Figure5. Track Car and Two-Dimensional Turntable

Because of thermal contraction at low temperatures, when the track car and two-dimensional turntable are designed, the main concern is how to avoid the fact deformation of materials at low temperatures blocks the bearing or between driving sliders and the track.

When designing process options, we take following measures:

1) Adopt single-row roller bearings and thin wall enclosure cross roller bearings. These kinds of bearings can adjust the gap between the bearing roller with internal and external bushings in order to achieve operational effects at low temperatures.

2) The little car has four wheels on the track: two are fixed, and two are floating with $3 \sim 5 \mathrm{~mm}$ expansion gap. Meanwhile, there is also some allowance for distance of car tracks, to avoid blocking the car in the track.

3) Add a friction surface in a different material on the contact area between the track and the car. Once they touch when running, this can reduce the friction coefficient as well as prevent scratches on the contact area.

When the slider part's temperature decrease from normal temperature to minimum temperature, a huge shrinkage deformation will take place. See Figure6. 


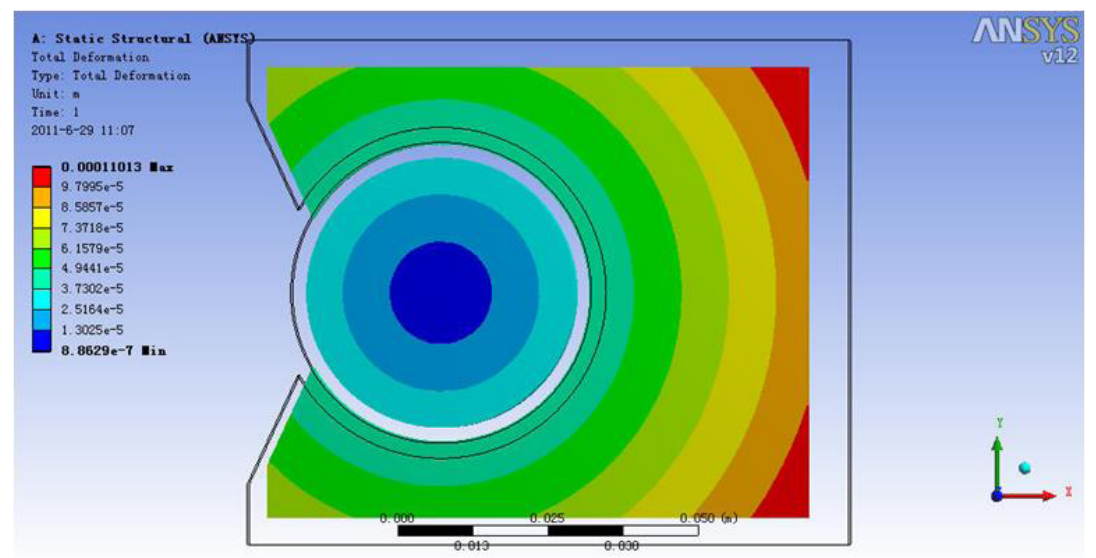

Figure 6. Deformation of Guiding Rail's sliders in Case of a Drop in temperature

In the figure, the black frame shows the original state before the drop in temperature, and colored areas indicate state after deformation. In terms of the drop in temperature and deformation, because the same stainless steel material is used, there is little deformation and the drop in temperature will not cause a block, and there is still a big moving space. The expansion reserved space can meet the requirements.

\subsection{Cable Lead-out Equipment}

Cables include power cord, electrical control lines, measuring equipment's signal lines, cables, etc. The working temperature of cables cannot be too low, so the cables require insulation and heating. Heating control is automatic in order to ensure the device's normal operation. Add fixed cables in cable bundles to bear tension and bending deformation, and to protect cables. There is also a shield layer and protection covers. Cable lead-out equipment is fixed in the middle of the guide rail, and extends horizontally (vertical to the track) to lead cables of the little car and test equipment. Near the lead-out equipment there is extension and retraction equipment which can meet cable's bending radius requirements, in order to avoid intertwining because of too long cables.

The extension and retraction of cables are controlled by drag chains made of stainless steel, in order to ensure normal operation and little air release at low temperatures.

\subsection{Monitor System}

The monitor system needs to control three axles for movements: walking (z), pitching (f), yaw (p). It needs to monitor technical parameters like the speed, location, etc. during loaded movement. To ensure the normal operation of the system, PMAC is employed to realize comprehensively control and monitor three motors and the whole movement system, to get real-time status of the whole movement system[3][4].

The monitor system has following main hardware:

1) 1 industrial control computer (including LCD)

2) PMAC and its accessories: control three servo motors, receive feedback signals to realize closed loop control, and have auxiliary I/O points for external control, etc;

3) Servo drive unit's electrical components (air switch, contactor, relay, related control's switch power, isolating transformer, and auxiliary $\mathrm{I} / \mathrm{O}$ wiring board, etc.);

4) Connector plugs of external motors, encoders and $\mathrm{I} / \mathrm{O}$ signals on the device side;

5) Cabinet and related control buttons;

6) The electric cabinet's design, assembly, wiring, debugging, etc.

Monitor software system is designed to control 3D motion simulator's walking, pitching, yaw and 
other equipment's movement, to control the speed and location of walking, pitching and yaw control, and to realize positioning movement of yaw, pitching and walking and reciprocating movement. Besides, amplitude and frequency of movement are adjustable.

\section{Heat Control Option}

To ensure stability, reliability and accuracy of the device in TV conditions, it's necessary to control the device's key heat sensitive components' stability. In this walking device, the motor, reducer and control cables belong to heat sensitive components. Heat control is required for these components, and the working temperature should be above $273 \mathrm{k}$, to ensure the operation reliability of the system.

To ensure that motors and reducers can be stable at $273 \mathrm{k}$ in TV conditions of $100 \mathrm{~K}$, key parts of motors and reducers are pasted with thin heating films, and covered with insulating materials. According to the motor structure, it is simplified into following simulation calculation method [5]. In Figure 7, it designs size and power of each part's thin heating film.

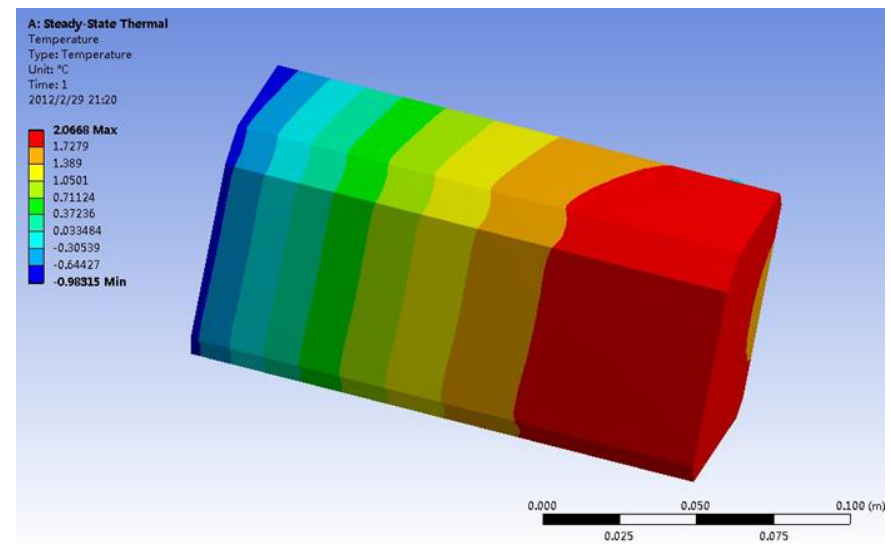

Figure7. Simulation Calculation of Motor's Heat Density

A $\mathrm{T}$ thermocouple is installed in the key area of every motor and reducer. The thermocouple is connected with thin heating films in series, PID intelligent temperature control device, and programmable DC power supply, to realize the closed loop control. Because of different heating boundary in each position and different power of heating films, 8 PID intelligent temperature control devices are used to control heat separately in 8 heating areas. Heating power cords and thermocouple connect exterior PID intelligent temperature control device by means of special connecting flanges to go through the tube wall by means. See principles as Figure8 below.

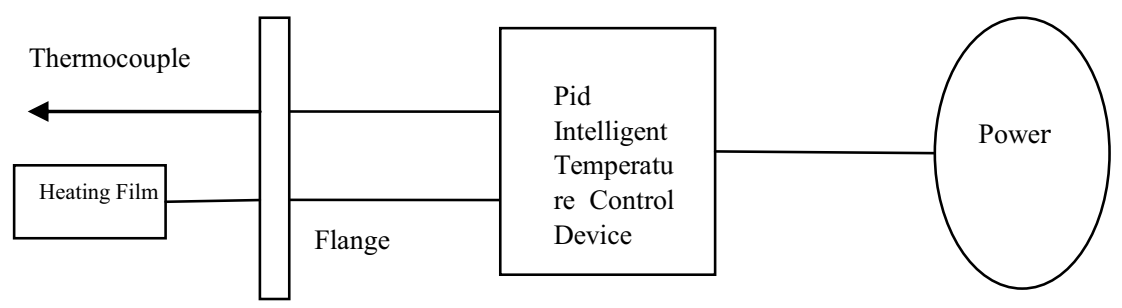

Figure8. Control Principle Chart 


\section{Test Application}

This device, after the successful development, has been used in spacecraft environment simulation tests for many times. The system's movement is steady and reliable. See real object in Figure 9. By installing laser measurement equipment in the two-dimensional turntable center, it adjusts the laser beam to go through the center of the ball of the facility and measure the direction accuracy of the device. The test results show that the accuracy exceeds $0.5^{\circ}$.

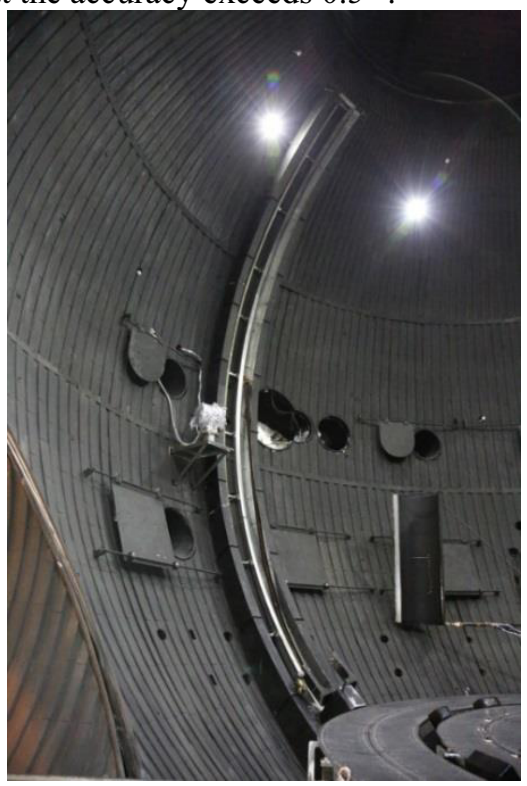

Figure 9. Photo of Large Arc-shape High-precision Walking Device

\section{Conclusion}

The successful development of Large Arc-shape High-precision Walking Device effectively solves problems in multi-angle and multi-direction measurement of optical images in large aircraft space environment simulation tests. Many test applications show that this device can move steadily and reliably without any blocking in TV conditions and with extremely accurate angle pointing.

\section{ACKNOWLEDGMENTS}

This work is supported by the National Natural Science Foundation of China (61671045).

\section{References}

1. XuGuohua, Tan Min. Status and trends of the mobile robot [J].Robot Technique and Application, 2001(3):6-14. (In Chinese)

2. Tanaka Y, ObaraS,SasakiA,etal.The effects of vibration in air and in ultrahigh vacuum on solid lubricated bearings[C]//IN: Proceedings of the 10th ESMATS.2003,524:49 56.

3. BorensteinJ.Control and kinematic design of multi-degree-of freedom mobile

4. Robots with compliant linkage [J].IEEE Transactions on Robotics and Automation,1995, 11(1):21-35.

5. ZhuangZhuo,YouXiaochuan,LiaoJianhui,et al. Finite element analysis and its application based on ABAQUS[M].Beijing:QinghuaUniversitiy Press,2009.(in Chinese) 